\title{
Temperature measurement in the flowing medium
}

\author{
Kamil Sedlák ${ }^{1, *}$, and Lenka Fialová ${ }^{1}$ \\ ${ }^{1}$ Doosan Škoda Power, s.r.o., Experimental research of flow, Tylova 1/57, 30128 Plzeň, Czech \\ republic
}

\begin{abstract}
The article deals with a brief description of methods of temperature measurements in a flowing water steam. Attention is paid to the measurement of pseudo static temperature by a single sealed thermocouple entering the flowing liquid through the flown-by wall. Then three types of probes for stagnation temperature measurement are shown, whose properties were tested using CFD calculations. The aim was to design a probe of stagnation parameters of described properties which can be used for measuring flow parameters in a real steam turbine. An important factor influencing the construction is not only the safe manipulation of the probe when inserting and removing it from the machine in operation, but also the possibility to traverse the probe along the blade length.
\end{abstract}

\section{Introduction}

From the viewpoint of measuring thermodynamic parameters of flowing water steam, it is possible to choose two different and basically correct approaches.

The principle of the first approach is to focus on static parameters measurement and, with the knowledge of velocity field character and the thermodynamic properties of the flowing medium, to calculate the total parameters. This process is well manageable for pressure measurements, but it fails for temperature measurement. Provided the measured temperature was really static, it would be necessary to guarantee the sensor movement in accordance with the flowing medium. In other words, if the static temperature is to be measured, it is necessary for the sensor (thermometer) to move not only at the same velocity, but also in the same direction in the three dimensional space as the flowing medium. From these requirements it is evident that such measurement is not possible in common technical practice.

In this situation the solution could be to measure the temperature by a stationary sensor and, to accept the fact that the measured temperature is neither static nor total but somewhere in between, or to attempt to measure the total temperature and add the so called recovery factor that will be defined later on in the text and which carries, in the standardized form, the information about how far the measured temperature is from the static one.

\footnotetext{
* Corresponding author: kamil.sedlak@doosan.com
} 
The motivation for this article were some unanswered questions, emerging mainly in relation to the analysis of measurement uncertainties. One of them was how big the error of temperature measurement is when the thermometer does not move with the flowing medium, but the measured temperature is considered static; what values the corrective coefficient (recovery factor) has and if it is possible to measure overall temperature in the flowing steam environment using the experience with measurement in flowing air.

The aim was to design a probe of total parameters which could be used for measurements in steam turbine LP parts. The probe must be constructed to meet the requirements for stagnation parameters measurement. It must be also possible to insert it and remove it from the turbine in operation through the round opening in the turbine casing designed for pneumatic probe measurement.

\section{Study description}

The influence of thermodynamic heating of the flowing medium depends on thermodynamic properties of the medium, specifically on Poisson constant, and also on Mach number. The following graph shows the influence of both mentioned parameters on the difference between stagnation and static temperature of the ideal gas with selected Poisson constants.

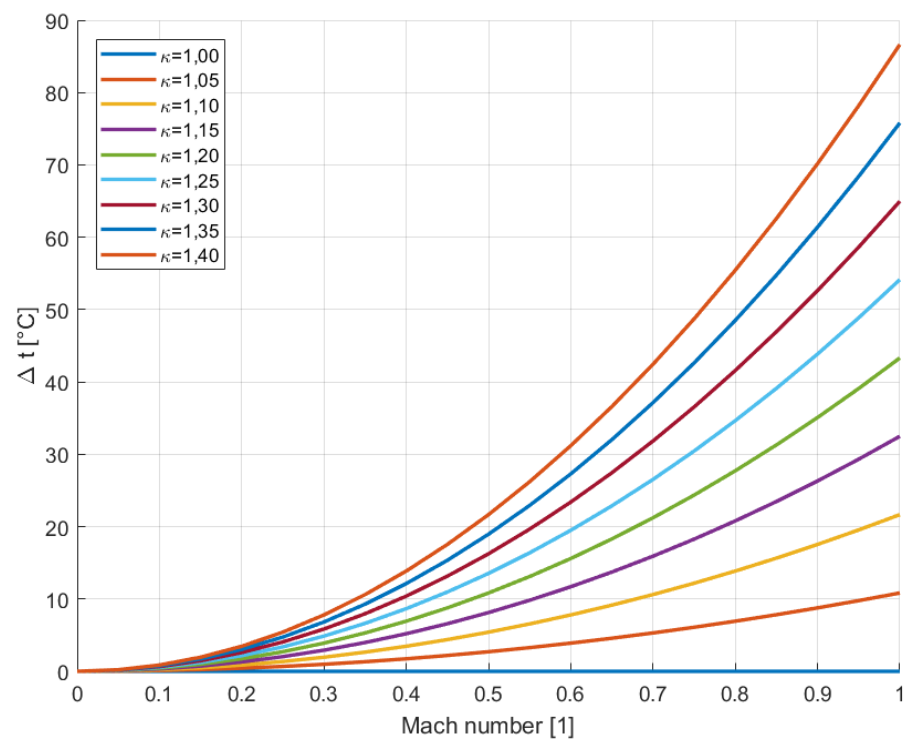

Fig. 1. Influence of Poisson constant and Mach number value on temperature difference.

The difference of both temperatures plotted in the graph above was derived from the equation given below.

$$
T / T_{0}=\left(1+(\kappa-1) \cdot M a^{2} / 2\right)
$$

The probe was designed to measure stagnation parameters in the flowing water steam environment for parameters calculated according to industrial formulation IAPWS IF97 and given in the following table.

Poisson constant value is $\sim 1.3$, which means that for Mach number 0.4 the difference between stagnation and static temperature is about $10^{\circ} \mathrm{C}$.

Two working media were considered: air, in the graph labelled "air" and ideal gas with water steam parameters given in the previous table, labelled as "ideal gas". 
Table 1. Flowing medium parameters.

\begin{tabular}{|c|c|c|}
\hline Quantity & Value & Units \\
\hline Specific thermal capacity for constant pressure & 1914.91 & {$[\mathrm{~J} / \mathrm{kg} / \mathrm{K}]$} \\
\hline Poisson constant & 1.3 & {$[1]$} \\
\hline Dynamic viscosity & $1.454 \mathrm{e}^{-05}$ & {$\left[\mathrm{Ns} / \mathrm{m}^{2}\right]$} \\
\hline Thermal conductivity & 0.0322 & {$[\mathrm{~W} / \mathrm{m} / \mathrm{K}]$} \\
\hline
\end{tabular}

In this work four geometrical variants of temperature measuring sensors were considered. Variant 0 is a common insulated sealed thermocouple or another cylindrical sensor passing through the flown-by wall and reaching with its active part the flowing medium, see the two following figures.
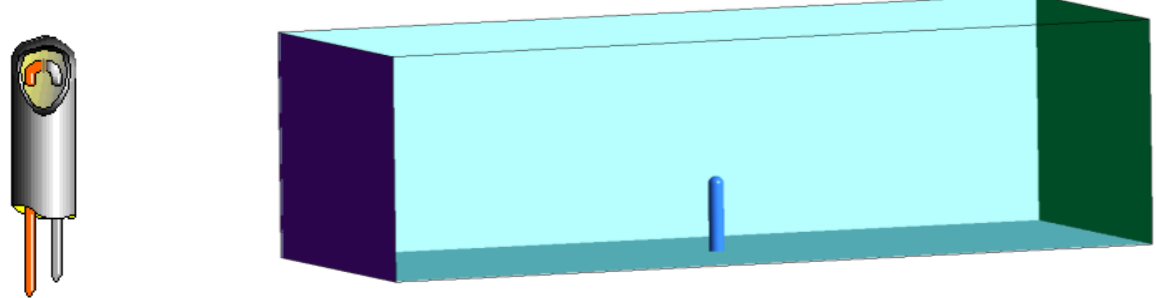

Fig. 2. Sealed thermocouple acc. [6], geometry task variant 0 .

The following three figures show the geometry of total parameters probes, variant 1 being the reference probe whose geometry was taken from [5]. Variant 2 and variant 3 are designed solutions where besides the resistance sealed sensor Pt1000 also total pressure take-off is fitted.
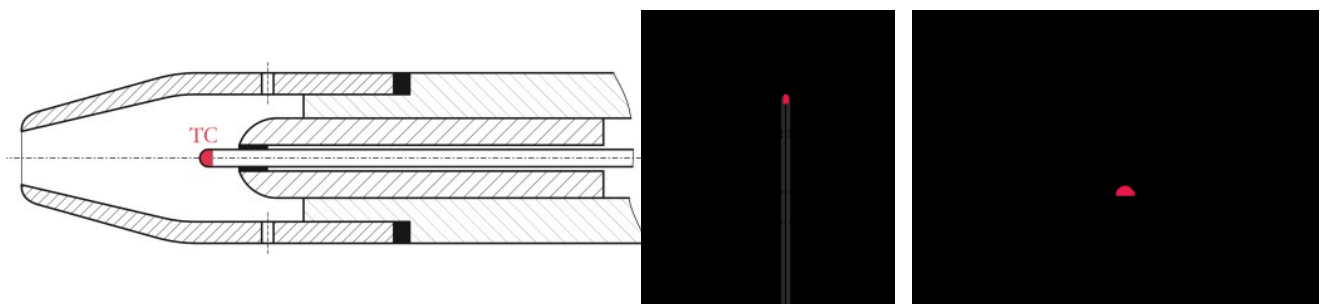

Fig. 3. Reference variant of probe 1 by [5], variant 2 and variant 3 .

For comparison of individual probe variants, the so called recovery factor was used that is defined by the following relation.

$$
r=\left(T_{\text {probe }}-T\right) /\left(T_{0}-T\right)
$$

It is a dimensionless coefficient that describes the standardized "distance" of measured temperature $T_{\text {probe }}$ from static temperature $T$, where value $1-r$ is the "distance" of measured temperature from total temperature $\mathrm{T}_{0}$.

The scope of Mach numbers was from 0.2 to 0.7 , for variant 2 the value 0.2 of Mach number was not considered and for variant 3 no results are available for $\mathrm{Ma}=0.7$.

In all cases several variants of circumferential and radial flow angle of the medium flowing on the probe were calculated. 


\section{Results}

Results are given in the following table where the influence of Mach number on recovery factor of all the above mentioned variants is shown.

Useful results were provided not only by variants of all three probes, but also by the cylindrical sensor, which was labelled as variant 0 . This is a common solution for temperature measurement in the flow part of the turbine, where Mach numbers can reach values from 0.2 up to 0.7 .

For the lowest Mach number, the recovery factor is 0.375 and with its growth recovery factor keeps dropping down to 0.325 . The temperature measured by the sealed cylindrical sensor is nearer the static temperature than the total temperature.

Referential probe characteristics (variant 1) is in the whole range more or less constant and near 1, which means that from the viewpoint of the total temperature measurement quality this is the best designed probe tested within this study. Similar results can be found in the original literature [5].

Out of the remaining two probes the better one seems to be the geometric variant 2 , whose characteristic is very near the referential variant, variant 3 characteristics change a lot dependent on Mach number; due to a low number of points it cannot be ruled out that inflection points appear in the probe characteristic.

Table 2. Recovery factor of individual variants for $\mathrm{Ma}=0.2-0.7$.

\begin{tabular}{|c|c|c|c|c|}
\hline \multirow{2}{*}{ Variant } & \multicolumn{4}{|c|}{ Mach number } \\
\cline { 2 - 5 } & 0.2 & 0.3 & 0.5 & 0.7 \\
\hline Variant 0 & 0.375 & 0.350 & 0.340 & 0.325 \\
\hline Variant 1 & 0.998 & 0.999 & 0.999 & 0.999 \\
\hline Variant 2 & - & 0.996 & 0.993 & 0.998 \\
\hline Variant 3 & 0.957 & 0.970 & 0.974 & - \\
\hline
\end{tabular}

In the following graph the difference is plotted of total and static temperatures depending on Mach number and Poisson constant. The curves are supplemented by points of temperature difference arising from CFD calculations for both media.

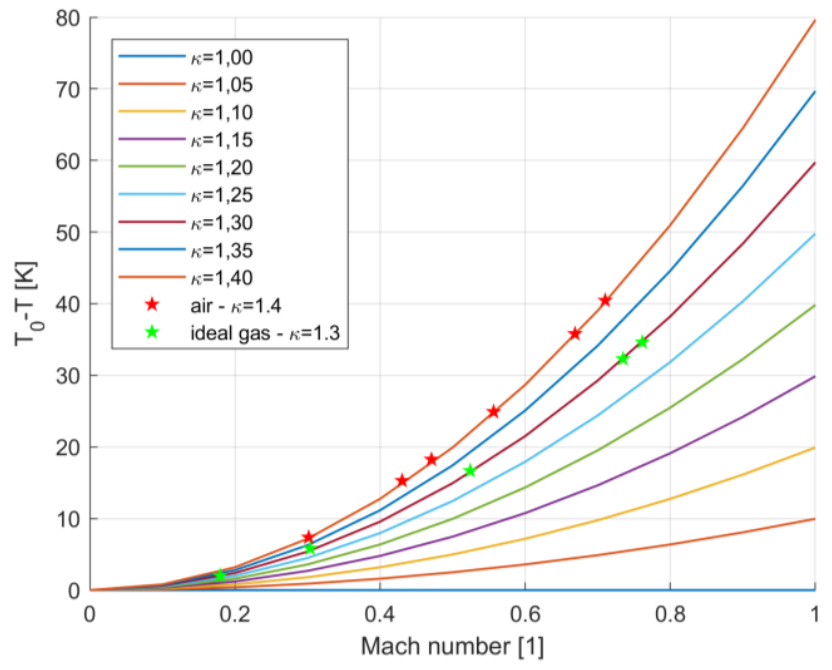

Fig. 4. Influence of Poisson constant and Mach number on temperature differences. 
It is evident from the graph that the points match the corresponding curves. It can be thus expected that the error caused by neglecting the influence of thermodynamic heating can be well described by the given relation (2). It means that it is possible to have a relatively large error in defining stagnation or static temperature for the given medium in relation to the Mach number values while neglecting or not knowing the recovery factor.

The error rate is further amplified by the increasing value of Poisson constant. It is necessary to keep in mind that for the real steam the isobaric or isochoric specific thermic capacity is not constant, thus even the iso-entropic exponent $\kappa$ cannot be considered a constant in the wide range of medium parameters. The value of exponent $\kappa$ can change dependent on the pressure and temperature in the range from $\sim 1.2 \div 1.7$. From the given graphs it is evident that the higher the $\kappa$ value, the larger error appears for the same Mach number in defining temperature. For $\kappa=1.7$ the error is $10^{\circ} \mathrm{C}$ for $\mathrm{Ma} \approx 0.3$.

\section{Conclusion}

Based on the study results the following was obtained:

- Characteristics of sealed cylindrical sensor, or thermocouple or resistance sensor,

- probe design that shows suitable properties for measuring stagnation parameters in flowing water steam in the steam turbine flow part,

- probe labelled as variant 2 fitted on the probe carrier for measurement behind the last stage of steam turbine LP part.

The probe was first tested in the aerodynamic tunnel. Measurement on the work was done in 2017 behind the last stage of LP part of the steam turbine of a large output whose owned and operated by ČEZ. Based on the experience gained the probe geometry was adjusted and fitted with a more accurate sensor for temperature measurement. Measurement on the steam turbine with the second generation probe has not been done yet.

Thanks belong to all involved, Doosan Škoda Power and the owner and operators of the ČEZ block and its employees for their helpfulness and assistance in the preparation and implementation of the measurements.

\section{References}

1. R.A. East, J.H. Perry, A Short Time Response Stagnation Temperature Probe, (1967)

2. M. Fernelius, S. Gorrell, Thermocouple Recovery Factor for Temperature Measurements in Turbomachinery Test Facilities, (2014)

3. A.H. Shapiro, The Dynamics and Thermodynamics of Compressible Fluid Flow, (1953)

4. C. Tropea, A. Yarin, J.F. Foss, Springer Handbook of Experimental Fluid Mechanics, (2007)

5. E. Winkler, Stagnation temperature probes for use at high supersonic speeds and elevated temperature, (1954)

6. http://www.capgo.com/Resources/Temperature/Thermocouple/Thermocouple.html 